\title{
RHEUMATOLOGY-OPHTHALMOLOGY COLLABORATIVEMANAGEMENT OF AUTO-IMMUNE OCULAR DISEASES IN A BRAZILIAN TERTIARY REFERRAL CENTER: A RETROSPECTIVE CASE SERIES
}

Leticia Cezar Araujo ${ }^{1, \star}$, Fernanda Lourenço Macagnani ${ }^{1}$, Camila Ávila Megda Cabianca ${ }^{1}$, Maria Thereza Gomes Caldeira ${ }^{1}$, Lívia Laila Soares Costa ${ }^{1}$, Daniela Pereira de Castro Alves ${ }^{1}$, Amanda Alexia Rodrigues Vieira ${ }^{1}$, João Marcello Fortes Furtado ${ }^{1}$, Rodrigo de Oliveira ${ }^{1}$, Rodrigo Luppino Assad ${ }^{1}$

1.Faculdade de Medicina de Ribeirão Preto, Ribeirão Preto (SP), Brazil.

*Corresponding author: let.cara@gmail.com

\section{BACKGROUND}

Identification of ocular manifestations in rheumatic diseases is important for precocious treatment and morbidity prevention, but also because it can serve as a marker for disease flares or even represent the first disease manifestation. Treatment usually involves immunosuppression, and the choice of agent, as well as management of adverse reactions and recognition of underlying systemic disease are factors that make a multidisciplinary approach necessary. Despite this, there is insufficient data to determine the structuring of multidisciplinary collaboration units or verify its benefit. This study aims to describe the clinical profile of patients diagnosed with autoimmune ocular diseases treated in a collaborative Rheumatology-Ophthalmology unit in a tertiary referral center and the potential benefits of multidisciplinary care.

\section{METHODS}

Retrospective chart analysis. Ethics approval was obtained from the Hospital das Clínicas da Faculdade de Medicina de Ribeirão Preto Ethics Committee.

\section{RESULTS}

Amongst the 145 included, most common referral origin was the ophthalmology's uveitis unit $(n=104,72.2 \%)$ and the main reason for referral was therapeutic support $(n=80,55.2 \%)$. Most common presentations were anterior uveitis $(n=70,48.3 \%)$, posterior uveitis ( $n=40,27.6 \%)$ and panuveitis $(n=17,11.7 \%)$. Most patients $(n=55,38.2 \%)$ had idiopathic conditions. Most commonly detected underlying systemic disease were Vogt-Koyanagi-Harada disease $(n=30,20.7 \%)$, inflammatory spondyloarthropathies $(n=14,9.6 \%)$ and Behçet's disease $(n=8,5.2 \%)$. Of all patients with identifiable systemic diseases $(n=31), 34.4 \%$ were diagnosed by the CROU team. Most patients were treated with synthetic disease modifying drugs ( $n=118,81.4 \%$ ) at some point, mainly methotrexate $(n=74,63.5 \%)$ and azathioprine $(n=54,45.8 \%)$. Twenty-six (17.9\%) patients used biologic agents, mostly antitumor necrosis factor $(n=24,16.0 \%)$.

\section{CONCLUSION}

Most cases of autoimmune ocular diseases in tertiary centers are idiopathic conditions, most commonly present with anterior uveitis and need immunosuppressive therapy. Although prospective, group-controlled studies are necessary to establish the benefits of multidisciplinary management in patient outcome, it seems to be helpful in recognizing underlying systemic diseases and optimizing immunosuppression adjustment.

\section{KEYWORDS}

Uveitis, Scleritis, Episcleritis, Multidisciplinary management, Collaborative unit. 